\title{
Radiografía de la presencia de la solidaridad, la cooperación, el desarrollo, y las ONGD en la prensa regional navarra
}

\author{
Hildegart GonZÁLEZ LuIS \\ Universidad de Navarra \\ hgonzal@unav.es
}

Recibido: $16 / 05 / 2012$

Aceptado: 18/12/2012

\begin{abstract}
Resumen
Este artículo analiza la presencia y el tratamiento de las informaciones relacionadas con solidaridad, el desarrollo, la cooperación y la acción de las ONGD en la prensa regional editada en Navarra. Los datos obtenidos del análisis cuantitativo y cualitativo permiten radiografiar la forma y el contenido de la cobertura de estos ítems, además de confirmar similitudes y diferencias al respecto entre la prensa regional, nacional e internacional. El estudio también constata la presencia de estereotipos sobre los PVD en las informaciones publicadas y el refuerzo que supone para la reputación de las ONGD la difusión de noticias sobre estas cuatro temáticas.

Palabras clave: Medios de comunicación, ONGD, cooperación, desarrollo, solidaridad

\section{X-Raying Presence from Solidarity, Cooperation, Development, and DNGO Action in the Regional Press Published in Navarra}

\begin{abstract}
This article analyzes the presence and treatment of information related to solidarity, development, cooperation and DNGO action, in the regional press published in Navarra.

The data obtained from qualitative and quantitative analysis allow x-raying the form and contents of covering these items, besides confirming related similarities and differences between the regional, national and international press. The study also verifies the presence of stereotypes about developing countries in the published information and the reinforcement for DNGO reputation that is meant with the diffusion of news about these four themes.
\end{abstract}

Keywords: Communication media, DNGO, cooperation, development, solidarity

Referencia normalizada

GONZÁLEZ LUIS, Hildegart (2013): "Radiografía de la presencia de la solidaridad, la cooperación, el desarrollo, y las ONGD en la prensa regional navarra”. Estudios sobre el mensaje periodístico. Vol. 19, Núm. 1, págs.: 147-164. Madrid, Servicio de Publicaciones de la Universidad Complutense.

Sumario: 1. Introducción. 2. Objetivos y metodología. 3. Rasgos formales. 4. Rasgos del contenido. 5. Conclusiones. 6. Referencias bibliográficas.

\section{Introducción}

Las nuevas tecnologías están generado un nuevo paradigma comunicativo que posibilita que las denominadas genéricamente 'fuentes informativas' puedan difundir sus mensajes a la sociedad sin que sus contenidos se sometan a un proceso de mediación por parte de los periodistas que trabajan en la prensa, la radio y la televisión (McQuail, 2000).

A pesar de la implantación paulatina de este nuevo paradigma comunicativo, el que le predecía no ha desaparecido, sino que en la actualidad ambos conviven llegando, en muchos casos, a una interacción meritoria de análisis profesional y académico (Orihuela, 2011). 
La existencia de ambos paradigmas comunicativos obliga a que la mayoría de las fuentes informativas apuesten por invertir en procesos de comunicación que les permitan seguir presentes tanto en los medios off line como en los medios on line.

Por ello es posible afirmar que, a pesar de la ampliación de los canales por los que la información fluye en el siglo XXI, para un porcentaje significativo de las fuentes informativas y de la sociedad, los medios impresos tradicionales siguen siendo en la actualidad un referente, un interprete, una ventana, que continua señalando, enmarcando, estructurando y explicando a través de su agenda aquellos hechos noticiosos meritorios de ser conocidos (McCombs, 1996).

De todas las metáforas empleadas para delimitar las funciones sociales de los medios englobadas en la teoría de la mediación, esta investigación se centra en la de "intérprete", principalmente, por la contribución que en su ejercicio los periodistas pueden realizar a la solidaridad y al entendimiento de los problemas mundiales (McQuail 1998: p. 387-391).

En pleno siglo XXI la afirmación 'lo que no se publica en los medios no existe', sigue siendo válida, más, si cabe, en informaciones relacionadas con la cooperación, la solidaridad, el desarrollo y la acción de las organizaciones no gubernamentales de desarrollo (ONGD).

La presencia de estos asuntos en los medios informativos, no sólo repercute y condiciona el conocimiento que la opinión pública tiene de estas realidades (Fundación Carolina: p. 28; Peredo: 1999; Nos: 2002), sino que llega a influir, incluso, en la cantidad y el destino de la ayuda al desarrollo y de emergencia gestionada por las instituciones públicas, tal y como se ha constatado en investigaciones internacionales realizadas al respecto (Van Belle; et al, 2004).

Las múltiples e interesantes iniciativas activadas por parte de las ONGD para comunicarse empleando las oportunidades que proporciona el paradigma on line (Ramil, 2012: web; Burgui, 2010; Soria, 2011) todavía no han logrado sustituir la influencia que ostentan los medios tradicionales.

\section{Objetivos y metodología}

Este artículo pretende analizar la presencia y el tratamiento que los periodistas realizan sobre las informaciones relacionadas con la solidaridad, el desarrollo, la cooperación y la acción de las ONGD en la prensa regional editada en Navarra. Con los datos obtenidos en esta 'radiografía' la investigación pretende poder refrendar o refutar las siguientes hipótesis:

a) Cuando la prensa navarra publica noticias de carácter internacional, tiende a destacar en ellas los aspectos más locales intrínsicos en dichas informaciones, de modo que su contenido se 'regionaliza'. A pesar de esta peculiaridad, la prensa navarra comparte con la prensa nacional e internacional algunos rasgos que definen cómo son las coberturas de los items analizados.

b) La imagen de los Países en Vías de Desarrollo (PVD) difundida por los diarios navarros sigue incidiendo en las carencias estereotipadas atribuidas a estos países.

c) Las noticias que publican ambos rotativos sobre los items analizados tienden a reforzar y consolidar el buen hacer y la reputación de las ONGD. 
El estudio se centra en la Comunidad Foral por ser la que, a pesar de la crisis y los recortes económicos, sigue encabezando los rankings que miden la contribución de las regiones españolas en materia de cooperación al desarrollo (Intermon-Oxfam, 2012).

La muestra de análisis la componen todas las unidades informativas que incluyen una o varias de las palabras claves, "items", (solidaridad, desarrollo, cooperación, ONGD) publicadas por Diario de Navarra y Diario de Noticias en sus ediciones impresas desde el 1 de septiembre de 2010, al 31 de agosto de 2011. El vaciado de los dos diarios aportó un total de 465 unidades periodísticas (UP) que cumplían los requisitos mencionados.

Las herramientas metodológicas utilizadas en esta investigación son el análisis de contenido cuantitativo y el análisis de contenido cualitativo (Bardin, 1986; Bericat, 1998; Wimmer, 1996).

Una vez diseñado el código de análisis cuantitativo, se realizó un pretes sobre un $10 \%$ de la muestra para evaluar y mejorar su eficacia. Tras incorporar a esta herramienta de análisis las mejoras sugeridas por el pretes, se aplicó a toda la muestra.

Los datos obtenidos permiten describir los rasgos formales que caracterizan la presencia de estos cuatro ítems en los diarios (secciones, tamaño, mes, día, etc.) y el tratamiento que reciben estos contenidos en las UP publicadas (fuentes empleadas, temáticas principales, protagonistas, etc.). La información obtenida del análisis cualitativo ha permitido complementar y enriquecer estos resultados a través del procedimiento, triangulación (Bericat, 1998: p. 111). A continuación se detallan las principales conclusiones obtenidas.

\section{Rasgos formales}

La primera afirmación que se deduce del análisis de contenido realizado constata que entre los dos periódicos navarros publican una media superior a una noticia diaria sobre los items analizados. Sin embargo, la presencia no es proporcional en los dos rotativos. Diario de Noticias ha publicado casi dos tercios (61\%) de las noticias difundidas sobre cooperación, desarrollo, solidaridad y ONGD, frente al 39\% de Diario de Navarra.

De estos datos se puede inferir que, en la prensa regional ${ }^{1}$, al igual que constatan estudios precedentes que analizaban esta cuestión en la prensa nacional e internacional, los diarios de corte centro derecha son menos propensos que la prensa de centro izquierda a publicar en sus páginas este tipo de temáticas (Murciano, 2010: p. 145).

En cuanto al año de publicación, 2010 concentra un porcentaje de noticias llamativo: el 58,8\%. Resultado que no deja de sorprender, ya que la muestra analizada sólo contaba con las noticias publicadas durante el último cuatrimestre de ese año: septiembre, octubre, noviembre y diciembre. Por el contrario, en 2011, a pesar de formar parte de la muestra un número superior de meses correspondientes a este año (de enero a agosto), el número de UP es inferior.

${ }^{1}$ Según sus estatutos, Diario de Navarra se puede clasificar en la tipología centro, derecha, mientras que Diario de Noticias puede ser etiquetado como de centro izquierda. Cfr. (Sarries; Casares, 2010: pp. 229-248). 
La primera hipótesis barajada para explicar este descenso fue considerar que durante los últimos cuatro meses de 2010 se produjo algún acontecimiento noticioso de alto impacto sobre estos items, hipótesis descartada tras analizar los datos obtenidos sobre las temáticas principales publicadas en ese periodo. También se descartó la hipótesis de que fuera la época navideña la que justificara una mayor presencia ya que, como se expondrá en párrafos posteriores, el porcentaje de noticias publicadas en diciembre desciende frente a los meses anteriores.

La hipótesis que se considera más realista apunta a una caída paulatina del número de UP publicadas sobre estos items, a pesar de que no es posible corroborar empíricamente tal afirmación, ya que para ello sería necesario realizar un estudio comparativo complementario sobre la evolución de la cobertura durante el año 2012.

\begin{tabular}{|l|c|}
\hline \multicolumn{2}{|c|}{$\begin{array}{c}\text { Tabla 1. Distribución de las UP } \\
\text { por mes. Elaboración propia }\end{array}$} \\
\hline Mes & Porcentaje \\
\hline Enero & $6,6 \%$ \\
\hline Febrero & $4,4 \%$ \\
\hline Marzo & $6,1 \%$ \\
\hline Abril & $8,3 \%$ \\
\hline Mayo & $2,4 \%$ \\
\hline Junio & $12,5 \%$ \\
\hline Julio & $14,5 \%$ \\
\hline Agosto & $12,7 \%$ \\
\hline Septiembre & $10,5 \%$ \\
\hline Octubre & $11,6 \%$ \\
\hline Noviembre & $5,3 \%$ \\
\hline Diciembre & $5 \%$ \\
\hline Total & $100 \%$ \\
\hline
\end{tabular}

Con respecto al mes de publicación se constata que julio, con un $14,5 \%$, y junio, agosto, septiembre y octubre, que giran en torno al $11 \%$ y $12 \%$, son los meses que dan mayor cabida a noticias sobre los items analizados. De estos datos se puede concluir que es durante la época estival cuando los medios impresos, con un descenso claro de la actividad noticiosa en los ámbitos político, económico y social, son más proclives a publicar informaciones sobre solidaridad, cooperación, desarrollo y ONGD para suplir el vacío informativo.

Otro de los factores que puede justificar el aumento en el volumen de noticias publicadas es el hecho de que en verano estas temáticas se adjudican en las redacciones con cierta frecuencia a los alumnos becarios, tal y como confirma el análisis cualitativo, quiénes probablemente sean más propensos que sus colegas con mayor experiencia profesional a publicar información confeccionada a partir notas de prensa ${ }^{2}$.

Los datos ponen de relieve que los lunes y los sábados son los días de la semana en los que es menos frecuente poder leer informaciones relacionadas con los cuatro items analizados, $6,1 \%$ y $8,1 \%$ respectivamente. Por el contrario, los miércoles y los domingos acumulan 4 de cada diez noticias publicadas, $25 \%$ y $17,3 \%$.

El bajo porcentaje de UP publicadas los lunes puede deberse a dos factores: la escasa - nula proactividad informativa durante los fines de semana por parte de las ONGD y entidades que informan sobre estos items; y el significativo porcentaje de páginas destinadas por parte de la prensa a cubrir informaciones deportivas.

Partiendo de lo dicho en el párrafo anterior, es razonable pensar que si las informaciones promovidas por las entidades que trabajan en solidaridad, desarrollo y cooperación llegan a las redacciones los lunes, o los martes, se publican a mitad de

2 El análisis cualitativo constata esta afirmación, ya que corrobora un incremento de nuevas firmas de periodistas durante esos meses, firmas que desaparecen en la mayor parte de los casos a partir de octubre. 
semana, causa que puede explicar el aumento de impactos que se produce durante los miércoles, una cuarta parte del total publicado.

Los domingos, con un $17,3 \%$ son el segundo día de la semana que más porcentaje de noticias acumula, hecho que no sorprende, puesto que en la práctica periodista es frecuente que, si los profesionales no disponen de espacio para publicar estas temáticas entre semana, las pospongan al domingo, cuando la presión informativa decae de forma significativa en las redacciones de los diarios, y ambos rotativos disponen, además, de un mayor número de páginas en las que es frecuente encontrar reportajes y entrevistas ${ }^{3}$.

Los datos obtenidos muestran que la mayor parte de noticias se publican en Sociedad $(40,6 \%)$. Estos resultados permiten establecer un paralelismo entre la prensa regional, nacional e internacional en lo que a ubicación de noticias respecta. Según un estudio coordinado por el profesor Murciano, también la prensa nacional e internacional tiende a publicar porcentajes significativos de informaciones relacionadas con las ONGD, la cooperación descentralizada y la lucha contra la pobreza en la sección Sociedad (Murciano, 2010: p. 171; 90). Sin embargo, ese mismo estudio pone de manifiesto que si se amplia el concepto de cooperación a los standares internacionales en la materia, más allá de lo que la opinión pública y los propios periodistas incluyen bajo este término paraguas, es la sección Internacional la que aglutina el mayor número de UP publicadas (Murciano, 2010: pp. 9-11).

Por el contrario, en la prensa regional navarra, sólo un 6,8\% de las UP analizadas se publicó en al sección Internacional. Local con un 21,7\% y Política, con un 12,3\% son, tras Sociedad, las secciones que más porcentaje de noticias acogen en sus páginas.

Desde este estudio se valora como positiva la presencia de los ítems analizados en casi todas las secciones. La complejidad de la cooperación, el desarrollo, las ONGD y la solidaridad requiere que las informaciones que sobre ello puedan publicarse no sean tratadas únicamente en una sección, puesto que se produciría un reduccionismo en el enfoque y en los contenidos difundidos. Su ubicación en diversas secciones propicia una complementariedad de perspectivas en su tratamiento, al tiempo que aumenta la posibilidad de llegar a un conjunto más amplio de la sociedad que, además, puede intuir e incluso percibir la transversalidad inherente a estos items.

Si bien todos los datos expuestos hasta el momento revelan conclusiones positivas, hay tres porcentajes que permiten apuntar handicaps: escasa presencia de estos items en portadas $(1,1 \%)$, en contraportadas $(0,2 \%)$ y en la sección de economía $(0,7 \%)$.

Sin lugar a dudas, la noticia es el género prioritario a la hora de tratar estas cuestiones, $76,1 \%$. También es relevante destacar que la entrevista $(7,2 \%)$ y el reportaje $(6,6 \%)$, géneros que permiten un análisis más amplio y profundo de las informaciones difundidas son, tras la noticia, los dos formatos más empleados para abordar estas cuestiones.

Sobre los géneros de opinión merece la pena destacar que la cooperación, la solidaridad, el desarrollo y la acción de las ONGD se tratan en un porcentaje significativo de columnas, $6,4 \%$, porcentaje muy superior al que destina la dirección de los diarios a estas cuestiones, ya que sólo $0,7 \%$ de las UP analizadas son editoriales. Por

3 Conclusión obtenida de la revisión completa de los contenidos y paginación de ambos rotativos durante cuatro de las semanas que forman parte de la muestra analizada. 
último, merece la pena destacar el escaso porcentaje de cartas al director publicadas sobre estos items, $0,4 \%$, dato que evidencia que, o bien la sociedad navarra no canaliza su sensibilidad sobre estas cuestiones a través de los formatos habilitados para que los lectores de la prensa expresen su opinión, o bien los diarios no suelen publicar con asiduidad las cartas recibidas sobre estos items.

Algo más de la mitad de noticias publicadas lleva la firma de los propios redactores $^{4}$ de los diarios analizados, y un $8,6 \%$ llevan la marca genérica del rotativo. No sorprende el escaso número de UP firmadas por corresponsales, $(0,9 \%)$, ya que estos rotativos no disponen de los recursos económicos y humanos necesarios para mantener corresponsalías, carencia que la dirección de los periódicos suele justificar, aludiendo al escaso interés que sus lectores tienen por la información internacional. Cuando la actualidad lo precisa, la información la obtienen a través de agencias de comunicación, como refrenda el 31,1\% de las UP analizadas.

Sorprende el escaso uso que las ONG realizan de los espacios disponibles en la prensa para difundir información, ya que sólo el 1,5\% de la UP analizadas lleva la firma de estas entidades.

En párrafos anteriores se mencionaba que entre los dos periódicos publican una media de algo más de una noticia diaria sobre estos items. De ellas, más del $60 \%$ son pequeñas: inferiores a media página $(47,8 \%)$, o breves $(12,9 \%)$. Es relevante destacar que más de un tercio acapara espacios amplios: superiores a media página $(26,5 \%)$; página completa $(9 \%)$ y doble página $(3,7 \%)$. El porcentaje publicado en página par $(48,5 \%)$, es casi idéntico al difundido en impar (47,8\%), siendo un $3,7 \%$ las UP que abarcan ambas caras.

La primera conclusión sobre la presencia de elementos gráficos en las UP es que casi un $66 \%$ cuenta con fotografía, siendo un 3,5\% de ellas las que además, van acompañadas de mapa o infografía. La interpretación de esos datos permite un análisis doble: la elevada presencia de elementos visuales en la mayoría de las informaciones incentiva la atracción de los lectores, lo que aumenta la posibilidad de que se adentren en el texto, pero al mismo tiempo, estos elementos disminuyen el espacio otorgado al contenido, muy necesario para explicar con acierto, los items analizados que, como ya se ha mencionado anteriormente, son complejos.

\section{Rasgos del contenido}

El estudio constata la variedad de protagonistas en las fotografías que ilustran las UP analizadas. Los beneficiarios, con un $20 \%$, son quienes están presentes en el porcentaje más elevado de fotografías, siendo ésta, en un 2,2\%, una presencia compartida con cooperantes sobre en terreno ${ }^{5}$.

${ }_{4}$ El análisis cualitativo evidencia que, en Diario de Navarra, María Jesús Castillejo es, con marcada diferencia, la periodista que más UP rubrica. En Diario de Noticias son tres los profesionales más proclives a redactar sobre estos ítems: Ana Ibarra, Guillermo Nagore y Miguel Turullols.

5 Es posible que el 6,6\% obtenido por la categoría 'varias de las tipologías anteriores' los beneficiarios estén presentes. 
Sin embargo, si analizamos los datos agrupando a los protagonistas en dos categorías, PVD y Países Desarrollados (PD) la prevalencia de actores que residen - proceden de los últimos es considerablemente superior, sumando entre personal de la ONG en sede (11\%), voluntarios $(6,6 \%)$, sociedad civil activa $(6,6 \%)$, cooperantes en terreno $(1,5 \%)$, políticos $(6,1 \%)$, artistas $(2,2 \%)$ y público asistente a eventos $(0,9 \%)$, casi un $35 \%$ de las UP.

En el 20\% de UP en las que las fotografías recogen a los beneficiarios, éstos aparecen sobre todo en grupo, ya que un $9,9 \%$ responden a 'varias de las posibles tipologías analizadas". Los niños, con un $8,1 \%$, son el siguiente beneficiario más presente, seguido por hombres, $4,2 \%$ y mujeres, $1,5 \%$.

En la mayoría de las fotografías analizadas en las aparece el beneficiario de la cooperación su imagen es negativa, $12,1 \%$. Es decir, aparecen en actitud pasiva, desvalidos, sufriendo, siendo ayudados, etc ${ }^{6}$. Porcentaje significativamente menor, 7,9\%, los recoge en actitud activa o sonrientes ${ }^{7}$. En un 3,6\% su imagen no evidenciaba ninguno de los rasgos enunciados, siendo tipificada como neutra.

La representación negativa del beneficiario no es exclusiva de las fotografías que ilustran las UP, sino que caracteriza también el modo en el que éstos aparecen reflejados en el contenido de las informaciones publicadas. En un 19,9\% de los textos analizados son descritos con atributos que conllevan connotaciones negativas (desnutridos, impuros, apestados ${ }^{8}$ ), porcentaje muy elevado, sobre todo, considerando que en el 67,3\% de las UP ni siquiera se les menciona. Sólo un 9,9\% de las UP analizadas aporta en su contenido una imagen positiva9.

En cuanto a los continentes, el más mencionado en las noticias es Europa (32,9\%), dato coherente con otros aportados a lo largo de la investigación que constata una vez más el reenfoque que los medios regionales hacen de unos items que, en esencia, tienen un carácter internacional. El segundo continente con más menciones en las UP es África (26,8\%), seguido de América (15,6\%), siendo el menos presente Asia, (5,9\%).

\begin{tabular}{|l|c|l|c|}
\hline \multicolumn{4}{|c|}{ Tabla 2. Temáticas principal y secundaria. Elaboración propia } \\
\hline Temática principal & Porcentaje & Temática secundaria & Porcentaje \\
\hline $\begin{array}{c}\text { Acciones de emergencia desarro- } \\
\text { lladas por ONG }\end{array}$ & $2,6 \%$ & $\begin{array}{c}\text { Acciones de emergencia desarrolladas } \\
\text { por ONG }\end{array}$ & $3,7 \%$ \\
\hline Proyectos de desarrollo en terreno & $8,8 \%$ & Proyectos de desarrollo en terreno & $7,2 \%$ \\
\hline Recortes en ayudas/ subvenciones & $3,9 \%$ & Recortes en ayudas/ subvenciones & $4,2 \%$ \\
\hline $\begin{array}{l}\text { Adjudicación de subvenciones } \\
\text { públicas }\end{array}$ & $4,2 \%$ & Adjudicación de subvenciones públicas & $2 \%$ \\
\hline Adjudicación ayudas de privadas & $2,2 \%$ & Adjudicación ayudas de privadas & $0,7 \%$ \\
\hline
\end{tabular}

${ }^{6}$ Cfr. Diario de Navarra (24-10-2010, p.11); (28-7-2011, p. 8); (27-07-2011, p.1); (5-9-2010, p.6); Diario de Noticias (31-1-2010, p.6).

7 Cfr.: Diario de Navarra (21-7-2010, p. 20); Diario de Noticias (15-9-2010, p. 10)

8 Sirvan de ejemplo las siguientes UP: Diario de Navarra (28-07-2011, p.8); (12-10-2001, p.48); Diario de Noticias (15-9-2010, p. 9)

9 Cfr: Diario de Navarra (24-10-2010, p 20); Diario de Noticias (10-6-2010, p.10) 


\begin{tabular}{|l|c|l|c|}
\hline $\begin{array}{c}\text { Desastres naturales + emergencias } \\
\text { humanitarias }\end{array}$ & $5,3 \%$ & $\begin{array}{l}\text { Desastres naturales + emergencias hu- } \\
\text { manitarias }\end{array}$ & $5,9 \%$ \\
\hline Aniversarios de ONGD & $0,9 \%$ & Aniversarios de ONGD & $0,2 \%$ \\
\hline Acciones de captación & $3,9 \%$ & Acciones de captación & $5,3 \%$ \\
\hline $\begin{array}{c}\text { Manifestaciones promovidas por } \\
\text { ONGD }\end{array}$ & $3,3 \%$ & $\begin{array}{l}\text { Manifestaciones promovidas por } \\
\text { ONGD }\end{array}$ & $0,4 \%$ \\
\hline Voluntariado & $12,5 \%$ & Voluntariado & $4,2 \%$ \\
\hline $\begin{array}{l}\text { Eventos que ponen en valor cul- } \\
\text { tura de PVD }\end{array}$ & $4,6 \%$ & $\begin{array}{l}\text { Eventos que ponen en valor cultura de } \\
\text { PVD }\end{array}$ & $1,5 \%$ \\
\hline $\begin{array}{c}\text { Recomendaciones a la sociedad } \\
\text { para cooperar }\end{array}$ & $1,1 \%$ & $\begin{array}{l}\text { Recomendaciones a la sociedad para } \\
\text { cooperar }\end{array}$ & $1,1 \%$ \\
\hline Inmigración & $0,9 \%$ & Inmigración & $1,1 \%$ \\
\hline $\begin{array}{c}\text { Profesionalización de las planti- } \\
\text { llas del sector }\end{array}$ & $1,8 \%$ & $\begin{array}{l}\text { Profesionalización de las plantillas del } \\
\text { sector }\end{array}$ & $0,7 \%$ \\
\hline Día mundial de & $1,1 \%$ & Día mundial de & $0,2 \%$ \\
\hline Comercio justo & $0,4 \%$ & Comercio justo & $1,8 \%$ \\
\hline Testimonios de los PVD & $3,3 \%$ & Testimonios de los PVD & $2,4 \%$ \\
\hline Premios recibidos por ONGD & $4,2 \%$ & Premios recibidos por ONGD & $1,1 \%$ \\
\hline $\begin{array}{c}\text { Objetivos de Desarrollo delMile- } \\
\text { nio }\end{array}$ & $3,5 \%$ & Objetivos de Desarrollo del Milenio & $3,7 \%$ \\
\hline Caso Urchueguía & $9 \%$ & Caso Urchueguía & $3,1 \% \%$ \\
\hline Aniversario de desastres naturales & $0,9 \%$ & Aniversario de desastres naturales & $0,4 \%$ \\
\hline Otras & $5,5 \%$ & Otras & $9,9 \%$ \\
\hline Secuestros & $4,2 \%$ & Secuestros & $1,3 \%$ \\
\hline Flotilla palestina & $3,1 \%$ & Flotilla palestina & $0,2 \%$ \\
\hline Violencia en PVD & $2,2 \%$ & Violencia en PVD & $1,1 \%$ \\
\hline $\begin{array}{l}\text { Hambrunas o problemas en PVD } \\
\text { prolongados en tiempo }\end{array}$ & $6,6 \%$ & $\begin{array}{l}\text { Hambrunas o problemas en PVD pro- } \\
\text { longados en tiempo }\end{array}$ & $1,3 \%$ \\
\hline Noticias sobre género/ mujer & $0,2 \%$ & Noticias sobre género/ mujer & $0,7 \%$ \\
\hline & $\mathbf{1 0 0 \%}$ & Total & $\mathbf{1 0 0 \%}$ \\
\hline Total & No hay temática secundaria & $0 \%$ \\
\hline
\end{tabular}

La temática principal más presente en las UP analizadas es el voluntariado, con un $12,5 \%$, dato que no sólo evidencia la elevada participación de la sociedad navarra en este tipo de acciones, sino que muestra cómo la prensa tiende a recoger frecuentemente estas informaciones en sus páginas.

La segunda temática más presente a lo largo del periodo analizado es el denominado 'caso Urcheguía', asunto que acapara el 9\% del total de UP. La elevada cobertura que la prensa regional otorga a un supuesto delito de adquisiciones ilegales de terrenos en PVD por parte de una ONG con sede en el País Vasco evidencia la relevante repercusión mediática que tienen las crisis de comunicación en las que se ve implicada alguna entidad del sector no lucrativo.

Los proyectos desarrollados en terreno promovidos por las ONGD, con un $8,8 \%$, son la tercera temática con mayor presencia en la muestra analizada, con tres puntos porcentuales de diferencia frente la categoría 'otros', con un 5,5\%, dato significativo, sobre todo teniendo presente la elevada variedad temática incluida en el código que queda reflejada en la tabla anterior. 
Resulta interesante destacar que, si se reagrupan las categorías recogidas en la tabla anterior bajo el criterio: temas que acontecen en PVD y temas que acontecen en PD se evidencia el enfoque marcadamente negativo (Moeller: 1999), incluso 'amenazante' (Díaz: p. 382), que poseen la mayoría de los hechos noticiados recogidos en el primer grupo, así como su inferioridad porcentual. Esta reagrupación constata que los medios regionales navarros tienden a publicar más UP sobre cooperación, desarrollo solidaridad y acciones de ONGD cuando éstas acontecen en un PD.

También es interesante destacar que un $14,6 \%$ de las UP versa sobre cuestiones económicas: adjudicación de subvenciones públicas $(4,2 \%)$; recortes en las ayudas $(3,9 \%)$; acciones de captación de ONGD (3,9\%); adjudicación de ayudas privadas $(2,2 \%)$; siendo mucho menor el porcentaje de noticias dedicadas al comercio justo $(0,4 \%)$. Los porcentajes de los temas mencionados, que agrupados se acercan al $15 \%$ de las UP, contrastan con la ya mencionada baja presencia en la sección de Economía $(0,7 \%)$, de los ítems analizados.

Por último, la triangulación de estos datos con los resultados obtenidos del análisis cualitativo confirma que la gran mayoría de las noticias que tratan sobre adjudicaciones de subvenciones públicas o privadas son consecuencia de notas informativas que las entidades donantes han remitido a los periodistas. Ello evidencia el interés de estas entidades por dar a conocer a la sociedad navarra las partidas concedidas.

La mayoría de las UP analizadas no tienen una temática secundaria $(34,6 \%)$, lo que confirma que casi dos tercios de las informaciones publicadas sobre los items analizados son monotemáticas. Tal y como queda recogido en la tabla anterior, la variedad en lo que al asunto secundario de la información respecta es también muy elevada, ya que en este caso, un $9,9 \%$ responden a un tema secundario que no se corresponde con ninguno de los propuestos en el amplio listado incluido en el código.

Proyectos de desarrollo en terreno (7,2\%); desastres naturales - ayuda humanitaria $(5,9 \%)$ y acciones de captación $(5,3 \%)$ son las tres temáticas secundarias más presentes en las UP analizadas.

Si se comparan los porcentajes sobre temática principal y secundaria expuestos en la tabla, se puede afirmar que los recortes en las ayudas; los desastres naturales y acciones de emergencias; las acciones de captación, la inmigración, el comercio justo; los Objetivos de Desarrollo del Milenio; y información sobre género son cuestiones que están presentes en las UP con más frecuencia como asunto secundario. Es decir, si bien es cierto que en ocasiones son la temática principal en algunas piezas analizadas, su presencia como asunto secundario los supera.

Los datos obtenidos en la investigación permiten afirmar que son algo menos de la mitad de las UP analizadas (49,3\%) las que mencionan alguna de las funciones que las ONGD pueden realizar (González, 2006: pp. 104-133). Este dato contrasta con el imaginario público al respecto, que parece atribuir a estas entidades a las que considera expertas, el monopolio en los cuatro ítems analizados. Apunta además que los medios impresos regionales reflejan con sus informaciones el amplio abanico de agentes de cooperación que interviene en este ámbito de acción, tal y como refrendan otros datos que se aportan en párrafos posteriores.

La principal función comunicativa desarrollada por las ONGD presente en las páginas de los diarios es la informativa (20,4\%), dato que refleja su relevante labor como 
fuentes autorizadas en los items analizados. Sin embargo, los datos obtenidos permiten afirmar que ese rol de fuentes se ejecuta principalmente desde la sede (14,5\%), ya que sólo en un 5,9\% de UP publicadas las ONGD aportan información desde el terreno.

También es interesante destacar que un 11,2\% de los casos, la presencia comunicativa de estas entidades en las UP pretende promover una sensibilización del lector. La labor de agentes de presión también adquiere presencia en un porcentaje significativo de noticias, aunque menor a los ya enunciados, $7,9 \%$. La función de captación de recursos económicos está presente en 7,2\%. La educación para el desarrollo y el voluntariado es la menos visible dentro del conjunto de UP analizadas, 2,6\%.

El último porcentaje aportado, puesto en correlación con el dato que mostraba que el voluntariado es la temática principal más frecuente en las UP que componen la muestra (12,5\%), requirió del análisis cualitativo específico de estas piezas informativas para determinar quién era el promotor de estas acciones de voluntariado. El análisis cualitativo constató el vasto y activo tejido social en Navarra, en el que colegios, asociaciones de vecinos, universidades, diócesis, y otro tipo de entidades promueven un amplio abanico de acciones de voluntariado que los dos diarios difunden ${ }^{10}$.

\begin{tabular}{|l|r|l|r|}
\hline \multicolumn{4}{|c}{ Tabla 3. Primera y segunda ONGD mencionada. Elaboración propia } \\
\hline $\mathbf{1}^{\mathbf{a}}$ ONGD mencionada & \multicolumn{1}{c|}{$\%$} & $\mathbf{2}^{\mathbf{a}}$ ONGD mencionada & \multicolumn{1}{c|}{$\%$} \\
\hline Acción contra el hambre & $2 \%$ & Acción contra el hambre & $0,4 \%$ \\
\hline Ingeniería sin fronteras & $0,9 \%$ & Ingeniería sin fronteras & $0 \%$ \\
\hline Intermón-Oxfam & $3,5 \%$ & Intermón-Oxfam & $1,5 \%$ \\
\hline Medicus Mundi & $4,8 \%$ & Medicus Mundi & $0,2 \%$ \\
\hline Setem & $0,7 \%$ & Setem & $0,2 \%$ \\
\hline Nuevo Futuro & $2 \%$ & Nuevo Futuro & \\
\hline CONGDN & $2,5 \%$ & CONGD & $0,7 \%$ \\
\hline Consorcio de ONGD & $1,3 \%$ & Consorcio de ONGD & $1,1 \%$ \\
\hline No se menciona ONGD & $42,5 \%$ & No se menciona ONGD & $80,5 \%$ \\
\hline ONG en general & $4,6 \%$ & ONG en general & $4,4 \%$ \\
\hline Alboan & $2 \%$ & Alboan & $0,4 \%$ \\
\hline Medicos del Mundo & $1,1 \%$ & Medicos del Mundo & $0,9 \%$ \\
\hline UNICEF & $3,3 \%$ & UNICEF & $0,2 \%$ \\
\hline Otras & $12 \%$ & Otras & $6,6 \%$ \\
\hline Vicente Ferrer & $1,5 \%$ & Vicente Ferrer & $0,2 \%$ \\
\hline Cruz Roja & $3,3 \%$ & Cruz Roja & $1,1 \%$ \\
\hline Manos Unidas & $2,4 \%$ & Manos Unidas & $0,2 \%$ \\
\hline Cáritas & $0,7 \%$ & Cáritas & $1,1 \%$ \\
\hline Pobreza Cero & $1,1 \%$ & Pobreza Cero & $0 \%$ \\
\hline MSF & $0,7 \%$ & MSF & $0,2 \%$ \\
\hline Organizaciones religiosas, diócesis & $1,3 \%$ & Organizaciones religiosas, diócesis & $0 \%$ \\
\hline
\end{tabular}

${ }^{10}$ Cfr.: Diario de Navarra (28-10-2010, p. 27); (22-9-2010, p. contra); (2-5-2011, p. 10); Diario de Noticias (1-10-2010, p. 13); 14-10-2010, p. 37); (10-12-2010, P. 28). 


\begin{tabular}{|c|c|c|c|}
\hline Barcelona Acció Solidaria & $1,1 \%$ & Barcelona Acció Solidaria & $0 \%$ \\
\hline ONAY & $0,9 \%$ & ONAY & $0 \%$ \\
\hline ASODECOM & $3,9 \%$ & ASODECOM & $0 \%$ \\
\hline Total & $100 \%$ & Total & $100 \%$ \\
\hline
\end{tabular}

El primer dato relevante que merece la pena destacar es el hecho de que en un $42,5 \%$ de las UP no se menciona ni al sector agrupado bajo la sigla ONGD, ni a título individual a ninguna de las entidades que lo componen. Este dato refrenda la conclusión obtenida en el párrafo anterior: las ONGD no ostentan un monopolio mediático en los ítems analizados en este estudio.

La tabla evidencia, además, el crisol de ONGD que a lo largo del año analizado han tenido presencia en los dos rotativos. Las entidades que aparecían mencionadas en tres o más UP se incluían en el código como categoría individual, llegando a sumar más de una veintena. Aquellas que tenían una presencia inferior a tres impactos anuales se aglutinan en 'otras ONGD', categoría que recoge un 12\% de las UP difundidas.

Las cuatro ONGD más mencionadas en primer lugar son: Médicos Mundi (4,8\%); ASODECOM, (3,9\%); Intermón-Oxfam, (3,5\%); y UNICEF y Cruz Roja, cada una de ellas con un $3,3 \%$ de las piezas publicadas.

UNICEF, Médicos Mundi y Cruz Roja son entidades que valoran la comunicación como necesaria, ya que disponen de profesionales dedicados a su gestión en sus delegaciones en Navarra. En el caso de Intermón-Oxfam, si bien la ONGD no dispone en la Comunidad Foral de una persona liberada para gestionar el ámbito comunicativo, es la ONGD de la Coordinadora Estatal que más recursos invierte en comunicación (CONGDE, 2009). Lo expuesto respalda la hipótesis defendida por otros investigadores: la valoración de la comunicación como herramienta clave y estratégica en estas entidades y la inversión de recursos personales y económicos en ella son factores que influyen en la presencia de las ONGD en los medios informativos y en el imaginario público (Balas, 2011; González 2006; Martín, 2009: web).

También es interesante analizar la significativa presencia de ASODECOM, la menos conocida de todas las presentes en el ranking, que sin embargo ocupa el segundo puesto. Mediante el análisis de contenido cualitativo de las informaciones en las que esta entidad es mencionada en primer lugar se constató que la crisis comunicativa por un supuesto delito de adquisiciones ilegales de terrenos en PVD fue la causa que elevó de forma significativa la presencia en la prensa regional de una entidad cuasi-desconocida hasta ese momento. El hecho de que sea la segunda ONGD más mencionada, concentrando además su presencia sólo un mes y medio del periodo anual analizado, apunta claramente a la elevada repercusión que tienen en los diarios las situaciones de crisis en este sector ${ }^{11}$.

Los datos obtenidos parecen confirmar que disponer de sede o delegación en Navarra es un factor que también repercute positivamente en la presencia de estas enti-

${ }^{11}$ La primera noticia que se publica data del 26 de febrero de 2011 y a última el 10 de abril de 2011. 
dades en los diarios de la Comunidad Foral, lo que confirma una vez más la 'regionalización' ya apuntada que realizan los medios. De hecho, todas las ONGD incluidas en la última tabla cumplen con este requisito, con dos únicas excepciones: ASODECOM y Barcelona Acció Solidaria. La presencia de ASODECOM en la prensa navarra viene motivada, como ya se ha expuesto, por la crisis en la que esta entidad se vio envuelta. En el caso de Barcelona Acció Solidaria (1,1\%), el análisis de contenido cualitativo confirma que todas las noticias en las que esta institución era mencionada versaban sobre el secuestro de tres de sus cooperantes en Mauritania. Lo expuesto hasta el momento parece confirmar que los rotativos navarros sólo informan sobre entidades con sede en otras regiones de España cuando lo noticiable adquiere carácter polémico o dramático.

La falta de 'posicionamiento de marca individual' que caracteriza a este sector también se constata en esta investigación, ya que un 4,6\% de la UP la primera alusión a estas entidades es genérica: ONGD. Éste es el segundo porcentaje más elevado, a sólo unas décimas del 4,8\% obtenido por Médicos Mundi. Por último, es interesante mencionar que en un 2,5\% de UP la primera mención recae en la Coordinadora de ONGD de Navarra (CONGDN).

\begin{tabular}{|l|r|}
\hline \multicolumn{2}{|c|}{$\begin{array}{c}\text { Tabla 4. Primer actor de cooperación } \\
\text { mencionado. Elaboración propia }\end{array}$} \\
\hline Primer actor de cooperación & \multicolumn{1}{c|}{$\%$} \\
\hline $\begin{array}{l}\text { Agencias de Naciones Unidas } \\
\text { (PNUD, ACNUR) }\end{array}$ & \multicolumn{1}{c|}{$13,6 \%$} \\
\hline ONG & $30,5 \%$ \\
\hline Coordinadora de ONGD Navarra & $2,2 \%$ \\
\hline Gobiernos Locales & $10,5 \%$ \\
\hline Gobierno Nacional & $4,2 \%$ \\
\hline AECI & $0,7 \%$ \\
\hline Organismos europeos & $2,2 \%$ \\
\hline Empresas & $3,1 \%$ \\
\hline Otros & $0,7 \%$ \\
\hline Ninguno & $27,9 \%$ \\
\hline Universidad & $0,7 \%$ \\
\hline Ciudadanía & $2,6 \%$ \\
\hline Misioneros & $1,3 \%$ \\
\hline & $\mathbf{1 0 0} \%$ \\
\hline
\end{tabular}

Unido a lo expuesto en párrafos anteriores, los datos obtenidos en esta tabla permiten apuntar que es muy posible que muchas de las UP publicadas sean consecuencia de acciones proactivas por parte de las ONGD, ya que en un $80,5 \%$ de las piezas analizadas no se menciona una segunda entidad y en un $4,4 \%$ de los casos en los que sí hay alusión a una segunda institución es el sector en conjunto el nombrado.

El análisis de contenido cualitativo confirmó dicha afirmación y reveló que si bien ésta es la dinámica prioritaria, excepcionalmente los diarios recogen noticias en las que se menciona a varias de estas entidades. En ellas se informa sobre los proyectos subvencionados por el Gobierno de Navarra o por Caja de Ahorros de Navarra, apareciendo el listado de las ONGD receptoras ${ }^{12}$.

El estudio verificó que existe diferencia entre el peso mediático que tienen las entidades que pertenecen a la CONGDN y aquellas que no forman parte de esta entidad asociativa. Los datos obtenidos muestran que el conjunto de las ONGD asociadas tienen una presencia mediática inferior $(25,2 \%)$ al de entidades no incluidas en la CONGDN $(32,2 \%)$.

${ }^{12}$ Cfr.: Diario de Navarra Diario de Noticias (1-3-2011, p. 9;) (29-6-2010, p. 10) 
El primer dato destacable es que en casi tres cuartas partes las UP publicadas sobre cooperación, desarrollo, solidaridad y ONGD están protagonizadas por un actor de cooperación, dato que no sorprende puesto que como puede evidenciarse en la tabla, esta investigación toma como referencia el concepto amplio de actor de cooperación al desarrollo.

Los datos aportados en páginas anteriores ya confirmaban que las ONGD no ostentan un monopolio mediático en las informaciones sobre los cuatro items investigados. Esta tabla lo corrobora, aportando porcentajes que permiten afirmar que, si bien es cierto que su protagonismo es el más elevado de todos los agentes de cooperación, (30,5\%), las agencias de Naciones Unidas, $(13,6 \%)$ y los Gobiernos locales $(10,5 \%)$ adquieren una presencia relevante en las UP difundidas.

El protagonismo que ostentan las entidades públicas en este tipo de noticias, si se aglutinan todas ellas en una única categoría, suponen un 17,3\%, tal y como puede comprobarse en la tabla anterior, lo que evidencia la significativa presencia de estos organismos en los diarios regionales como promotores de desarrollo, cooperación y solidaridad.

Es relevante mencionar que, en la muestra analizada, las empresas $(3,1 \%)$ tienen una visibilidad mediática mayor como agentes de cooperación que la propia CONGDN $(2,2 \%)$. También es preciso desatacar la escasa presencia de la Universidad como primer agente de cooperación, sobre todo considerando que Navarra cuenta con tres universidades. Sólo lo es en un $0,7 \%$ de las UP analizadas. El hecho de que la ciudadanía sea el actor principal en un $2,6 \%$ de los casos, y los misioneros en un $1,3 \%$, son datos que constatan la fortaleza social, confirmada

\begin{tabular}{|c|c|c|}
\hline Fuente & Principal & Secundaria \\
\hline$\overline{\text { ONGD }}$ & $23,7 \%$ & $12,1 \%$ \\
\hline Periodista & $12,3 \%$ & $5 \%$ \\
\hline Fuentes oficiales & $28,9 \%$ & $9,2 \%$ \\
\hline Ciudadanos & $2,9 \%$ & $1,5 \%$ \\
\hline Beneficiarios & $2,6 \%$ & $2,6 \%$ \\
\hline Coordinadora & $3,1 \%$ & $1,5 \%$ \\
\hline Otros & $11,6 \%$ & $5,5 \%$ \\
\hline Cooperantes & $8,1 \%$ & $7,2 \%$ \\
\hline No mención fuentes & $6,8 \%$ & $55,3 \%$ \\
\hline Total & $100 \%$ & $100 \%$ \\
\hline
\end{tabular}
ya por diversos datos obtenidos en esta investigación.

En lo que respecta a la relación fuentes informativas - periodistas de medios (Bernabé, 2001; Cáceres, 2011: pp. 303-324), es relevante destacar que, a pesar de que los datos obtenidos analizados desde las tipologías empleadas en esta investigación permiten afirmar que las fuentes oficiales, con un $28,9 \%$ del total, son los interlocutores más utilizados por parte de los periodistas al redactar sus noticias, el sector de las ONGD en conjunto, aglutinando todas sus tipologías, supera significativamente dicho porcentaje. Las ONGD, con $23,7 \%$, los cooperantes con un $8,1 \%$ y la CONGDN con un $3,1 \%$ suponen un $34,9 \%$ de las informaciones analizadas siendo, en conjunto, la fuente prioritaria más empleada por los periodistas navarros al informar a sus lectores sobre cooperación, desarrollo solidaridad y la acción de estas entidades. Estos datos confirman que los periodistas navarros otorgan al sector de las ONGD, autoridad y credibilidad para informar sobre los items analizados, aunque esa autoridad y credibilidad no es exclusiva, sino compartida con las fuentes oficiales. 
Los resultados muestran que el porcentaje de ocasiones en las que las ONGD son fuentes informativas a título individual $(23,7 \%)$, es siete veces superior a las ocasiones en las que el sector habla con voz única a través de la Coordinadora $(3,1 \%)$. Se puede afirmar que las ONGD siguen apostando por acudir a los medios de comunicación de forma particular, sin priorizar que sea su órgano asociativo el que ejerza como interlocutor del sector, desoyendo recomendaciones que al respecto plantean los periodistas (CONGDE, 2007).

También es interesante comparar la elevada presencia de testimonios de cooperantes $(8,1 \%)$ frente al de los beneficiarios de los proyectos $(2,6 \%)$. Estos datos permiten respaldar los planteamientos de quienes reclaman un mayor compromiso y esfuerzo para que los beneficiarios de estas entidades sean fuentes informativas directas, pretensión que las propias entidades del sector suscriben ${ }^{13}$.

En cuanto a las fuentes secundarias, cabe destacar que en un 55,3\% de las noticias analizadas no se menciona ninguna fuente que complemente a la principal, es decir, no existe ninguna segunda cita directa o indirecta. Si se une este dato al elevado porcentaje de noticias en las que el sector de las ONGD y las instituciones oficiales son empleadas como primera fuente (lo que apunta a una proactividad informativa por parte de estas entidades ya mencionada en párrafos anteriores), se puede concluir que el periodista otorga credibilidad a las mismas, no siendo práctica habitual la confrontación con otros interlocutores de la información facilitada.

La última conclusión relevante que se deduce de esta tabla es que, al igual que sucedía al analizar en quién recae el rol de fuente prioritaria, el sector de las ONGD en conjunto es también la fuente secundaria más empleada. Si se suman los porcentajes en los que ONGD, cooperantes y Coordinadora son fuente secundaria, se obtiene un $20,8 \%$, porcentaje que duplica en este caso al uso que los periodistas hacen de las fuentes oficiales $(9,2 \%)$.

A pesar de que la gran mayoría de las 456 UP analizadas no se incentiva a la sociedad navarra a participar en el campo de la cooperación, un 29,8\% de las informaciones sí fomentan esa participación. En análisis de contenido cualitativo confirma que dicha participación se promueve esencialmente a través de los testimonios de voluntarios o cooperantes que han regresado de sus estancias, o bien a través de testimonios de voluntarios que colaboran con las ONGD en Navarra ${ }^{14}$.

El análisis constata que en la mayoría de las UP analizadas (62,7\%) no se recogen en los textos la participación de la sociedad en ámbito de la cooperación, el desarrollo y la solidaridad. Sin embargo, en un $37,3 \%$ de ellas, sí que se evidencia dicha participación canalizada principalmente en dos tipologías: $10,7 \%$ de las informaciones hacen referencia a participación a través de aportaciones económicas; y en un porcentaje casi idéntico, un 10,3\%, en actividades de voluntariado sobre el terreno.

${ }^{13}$ El protagonismo, la interlocución directa de los habitantes de los PVD está presente en el Código de conducta de la Coordinadora.

${ }^{14}$ Cfr.: Diario de Noticias (16-6-2010, 8); (2-1-2011, p. 9); Diario de Navarra (7-8-2010, p. 25); (3-9-2010, p. 25) 
Estos datos permiten concluir que todavía los medios de comunicación impresos, cuando reflejan la participación de la sociedad en temas de cooperación, desarrollo y solidaridad, siguen difundiendo las dos modalidades tradicionales: voluntariado y aportación económica. Mucho menor es el porcentaje de UP en las que la participación ciudadana se visibiliza por los medios a través de la presencia en manifestaciones $(3,3 \%)$, en acciones de sensibilización, o educación para el desarrollo $(3,5 \%$, en ambos casos), o en comercio justo, donde el porcentaje es casi insignificante $(0,7 \%)$.

En lo referente a la imagen que se aporta de la primera ONGD mencionada, destaca el dato de que un 39,3\% de las noticias que hablan directamente de alguna ONGD lo hacen de manera positiva, dato que constata la buena reputación que estas entidades siguen teniendo en el espectro mediático, ya que sólo en un 1,8\% de los textos informativos, la imagen difundida sobre ellas es negativa. Por último, es preciso matizar en este caso que en un 53,3\% de las UP analizadas no se aporta ninguna imagen de las ONGD.

Sobre la imagen publicada de los profesionales de las ONGDs se puede afirmar que está no existe en el 53,7\% de las UP y en casi un $40 \%$ de las noticias en las que sí hay imagen, ésta es positiva. La imagen negativa de los profesionales aparece en el 3,3\%. Estos datos apuntan que aunque el sector goza de buena reputación mediática, las realidades que pueden disminuirla se concentran con mayor frecuencia en prácticas de sus profesionales. El análisis cualitativo detectó que, la mayoría de estas UP trataban sobre el 'caso Urcheguia', mostrando además que los periodistas, ante situaciones polémicas como la citada, no transmiten una imagen negativa de la ONGD, sino que concentran sus criticas en una persona 'cooperante', que en este caso, era también la responsable de la entidad.

En cuanto a la imagen de la cooperación en general, ésta está presente en casi tres cuartas partes de la muestra analizada, porcentaje superior al de las UP donde se aporta imagen de las ONGD y de los cooperantes. En más de la mitad de las informaciones $(55,7 \%)$ la imagen de la cooperación es positiva. Sin embargo, el porcentaje de piezas informativas en las que la imagen de cooperación es negativa, es muy superior $(13,4 \%)$ a los mencionados sobre ONGD, o sus profesionales. El cualitativo constata que en la mayoría de los casos el cuestionamiento sobre la eficacia de la cooperación es el aspecto que genera dicha imagen.

Los datos aportados permiten concluir que el ámbito de la cooperación goza de buena reputación mediática, aumentando ésta en las UP que tratan sobre ONGD o sobre cooperantes, y disminuyendo cuando se alude a la cooperación de forma genérica.

\section{Conclusiones}

La investigación corrobora que existen algunas similitudes en los rasgos que definen las coberturas que la prensa regional, nacional e internacional realiza sobre la cooperación, el desarrollo, la solidaridad y la acción de las ONGD. Las principales son: mayor propensión de los diarios de centro - izquierda, frente a los de centro - derecha a publicar en sus páginas este tipo de temáticas; y mayor presencia de estos ítems en las secciones de Sociedad. 
Se puede confirmar la regionalización que los dos rotativos navarros realizan de items con un marcado carácter global. El hecho de que el voluntariado sea la temática más presente como asunto principal; la tendencia a publicar más UP sobre cooperación, desarrollo solidaridad y acciones de ONGD cuando éstas acontecen en un PD, y/o tienen como protagonistas a habitantes de PD; el uso de estas entidades (principalmente de las que tienen sede en Navarra) como fuentes y la publicación de la mayoría de las UP en secciones con un marcado carácter local son, entre otros, algunos de los argumentos aportados en el artículo que lo avalan.

La investigación evidencia que la imagen de los PVD difundida en la prensa navarra incide en las carencias estereotipadas que se les atribuye a estos países. La escasa presencia de los beneficiarios de la cooperación en las UP y su representación negativa en la mayor parte de los casos, unido a la clara connotación dramática de las temáticas noticiadas que acontecen en estos países son los dos argumentos más relevantes que refrendan esta conclusión.

La investigación confirma que las ONGD no ostentan el monopolio mediático en los items analizados. Sin embargo, son, como sector, la fuente informativa más empleada por los profesionales en este tipo de coberturas, realidad que confirma la autoridad y credibilidad que el gremio periodístico les otorga. Si a ello se suman los bajos porcentajes de noticias publicadas en las que la imagen de la cooperación, de sus cooperantes o las ONGD es negativa, se puede afirmar que la presencia de noticias sobre los items analizados en los rotativos regionales contribuye a reforzar su reputación.

\section{Referencias bibliográficas}

BALAS LARA, Monserrat (2011): La gestión de la comunicación en el Tercer sector. Madrid, Esic.

BARDIN, Laureance (1986): Análisis de contenido. Madrid, Akal.

BERICAT, Eduardo (1998): La integración de los métodos cuantitativo y cualitativo en la investigación social. Significado y medida. Barcelona, Ariel.

BERNABÉ, Javier (2001): 'Las áreas de comunicación de las ONGD y el trabajo periodístico', Revista española de Cooperación y Desarrollo, $\mathrm{n}^{\circ}$ 7, pp. 127-141

BURGUI, Teresa (2010): 'Qué oportunidades ofrece la nueva cultura digital a las ONGD en su condición de entidades educadoras', en ERRO, Javier y BURGUI, Teresa (coords.): Comunicando para la Solidaridad y la cooperación, como salir de la encrucijada. Pamplona, Foro Comunicación, Educación y Ciudadanía.

CONGDE (2009): Informe de la Coordinadora de ONG para el desarrollo-España sobre el sector de las ONGD 2009. Madrid, CONGDE.

CONGDE (2007): Los medios de comunicación y a las ONGD: situación actual y retos, Madrid. CONGDE.

CÁCERES ZAPATERO, María Dolores (2011): "La construcción social de la realidad: el papel de los profesionales en el establecimiento de la agenda temática". 
Estudios sobre el mensaje periodístico. Vol. 17, núm. 2, pp.: 303-324. Madrid, Servicio de Publicaciones de la Universidad Complutense.

DÍAZ ARIAS, Rafael (2008): "La representación del mundo en los informativos de televisión, Estudios sobre el mensaje periodístico". Estudios sobre el mensaje periodístico. Vol. 14, pp. 363-384, Madrid, Servicio de Publicaciones de la Universidad Complutense.

FUNDACIÓN CAROLINA (2010): Barometro de la Fundación Carolina 2010. América latina y la cooperación al desarrollo en la Opinión pública española. Madrid, noviembre, Fundación Carolina - CIS.

GONZALEZ, Hildegart, (2006): Estrategias de comunicación en las ONG de desarrollo. Madrid, Cideal.

INTERMON OXFAM (2012): La realidad de la ayuda 2011. Una evaluación independiente de la ayuda y las políticas de desarrollo en tiempos de crisis, en: http://www.intermonoxfam.org/sites/default/files/documentos/files/Realidad_de_la ayuda_2011.pdf

MARTIN, Gema et al (2009): Así nos ven. ¿Qué sabemos y cómo valoramos a las ONGD?, en: http://www.coordinadoraongd.org/uploads/documentos/20100706 _130710_Asi_nos_ven_web_low.pdf

McCOMBS, Maxwell (1996): "Influencia de las noticias sobre nuestras imágenes del mundo", en BRYANT, Jennings y ZILLMANN, Dolf: Los efectos de los medios de comunicación. Investigaciones y teorías. Barcelona, Paidós, pp. 13-34.

McQUAIL, Denis (2000): Introducción a la teoría de la comunicación de masas (3º edición). Barcelona, Paidos.

McQUAIL, Denis (1998): La acción de los medios. Los medios de comunicación y el interés público. Buenos Aires, Amorrortu.

MOELLER, Susan (1999): Compassion fatigue. New York, Routledge.

MURCIANO, Marcial (2010): La prensa y la cooperación internacional Cobertura de la cooperación para el desarrollo en nueve diarios europeos. Sevilla-Zamora, Comunicación Social.

NAVAS, Alejandro y GONZÁLEZ, Hildegart (2010): "Los medios de comunicación en Navarra”, en: SARRIES SANZ, Luis y CASARES GARCÍA, Esther (coords.): Sociología y Sociedad en Navarra. Una visión del cambio. Pamplona, Navarra, Sahats Servicios Editoriales, pp. 229-248.

NOS ALDAS, Eloísa (2002): Medios periodisticos, cooperación y acción humanitaria. ¿Relaciones imposibles?. Barcelona, Icaria.

ORIHUELA, José Luis (2011): 80 claves sobre el futuro del periodismo. Madrid, Anaya multimedia.

PEREDO POMBO, José María (1999): Opinión pública y desarrollo. Madrid, Universidad Europea CEES. 
RAMIL FERREIRO, Xosé (2012): \#ParadigmaTIC@s Comunicación y cultura digital en las ONG de Desarrollo: http://desycom.files.wordpress.com/2012/02/culturadigital.pdf. Madrid, CONGDE.

SORIA IBAÑEZ, María del Mar (2011): La comunicación en las ONGD españolas: la influencia de internet en el modelo de relaciones con los públicos. Tesis doctoral. Málaga, Universidad de Málaga.

VAN BELLE, Douglas A. et al. (2004): Media, Bureaucracies, and Foreign Aid : A Comparative Analysis of United States, the United Kingdom, Canada, France and Japan. USA, Palgrave Macmillan.

WIMMER, Roger D. y DOMINIC, Joseph R. (1996): La investigación cientifica en los medios de comunicación. Una introducción a sus métodos. Barcelona, Bosch. 